\title{
NLRP3 inflammasome activation in gestational diabetes mellitus placentas is associated with hydrogen sulfide synthetase deficiency
}

\author{
WEI WU ${ }^{1 *}$, QING-YING TAN ${ }^{2 *}$, FANG-FANG XI ${ }^{1}$, YUN RUAN $^{2}$, JING WANG $^{2}$, \\ QIONG LUO $^{1}$, XIAO-BING DOU ${ }^{3}$ and TIAN-XIAO HU ${ }^{2,3}$ \\ ${ }^{1}$ Department of Obstetrics, Women's Hospital School of Medicine Zhejiang University, Hangzhou, Zhejiang 310006; \\ ${ }^{2}$ Department of Endocrinology, Chinese PLA 903rd Hospital (Former Chinese PLA 117th Hospital), Hangzhou, Zhejiang 310013; \\ ${ }^{3}$ School of Life Sciences, Zhejiang Chinese Medical University, Hangzhou, Zhejiang 310053, P.R. China
}

Received August 4, 2021; Accepted October 7, 2021

DOI: $10.3892 /$ etm.2021.11017

\begin{abstract}
The placenta may play a key role in the activation of inflammation and initiation of insulin resistance (IR) during gestational diabetes mellitus (GDM) pathogenesis. Interleukin (IL)-1 $\beta$ and IL-18, regulated by NLR family pyrin domain containing-3 (NLRP3) inflammasome, are important inflammatory cytokines in the initiation of maternal IR during GDM. However, the mechanism responsible for the regulatory of NLRP3 inflammasome in placenta remains unknown. Hydrogen sulfide $\left(\mathrm{H}_{2} \mathrm{~S}\right)$ exerts anti-inflammatory function partially via suppressing the activation of the NLPR3 inflammasome. The present study aimed to investigate the role of NLRP3 inflammasome, $\mathrm{H}_{2} \mathrm{~S}$ synthetase cystathionine- $\gamma$-lyase (CSE) and cystathionine- $\beta$-synthetase (CBS) in placenta in the pathogenesis of GDM. Clinical placenta samples were collected from pregnant women with GDM $(\mathrm{n}=16)$ and healthy pregnant women at term $(n=16)$. Western blot analysis was performed to detect the protein expression levels of NLRP3, cleaved caspase-1, CBS and CSE in the placenta samples. Pearson's correlation analysis was performed to assess the correlation between NLRP3 inflammasome and $\mathrm{H}_{2} \mathrm{~S}$ synthetase. Human placental cells were cultured and treated with different concentrations of NaHS $(0,10,25$ and $50 \mathrm{nmol} / \mathrm{l})$ or L-cysteine $(0$, $0.25,0.50$ and $1.00 \mathrm{mmol} / \mathrm{l})$. In addition, western blot analysis was performed to detect the protein expression levels of NLRP3 and cleaved caspase-1, while ELISA was performed to measure the production of IL-1 $\beta$ and IL-18 in the culture media. The results demonstrated that the expression levels of NLRP3 and cleaved caspase-1 increased, while the expression
\end{abstract}

Correspondence to: Dr Tian-Xiao Hu or Professor Xiao-Bing Dou, School of Life Sciences, Zhejiang Chinese Medical University, 548 Binwen Road, Hangzhou, Zhejiang 310053, P.R. China

E-mail: hutianxiao2005@163.com

E-mail: xbdou77@163.com

*Contributed equally

Key words: gestational diabetes mellitus, hydrogen sulfide, NLR family pyrin domain containing-3 inflammasome, placenta levels of CBS and CSE decreased in the placenta samples. In addition, the expression levels of NLRP3 and cleaved caspase-1 were inversely correlated with the expression levels of CBS and CSE. Notably, NaHS and L-cysteine significantly suppressed the expression levels of NLRP3 and cleaved caspase-1, and the production of IL-1 and IL-18 in human placental cells. Taken together, the results of the present study suggest that $\mathrm{H}_{2} \mathrm{~S}$ synthetase deficiency in placenta may contribute to excessive activation of NLRP3 inflammasome in GDM.

\section{Introduction}

Gestational diabetes mellitus (GDM) is a form of diabetes first recognized during pregnancy, which is characterized by glucose intolerance and insulin resistance (IR) (1). Epidemiological studies have reported that GDM affects $\sim 15.5-19.9 \%$ of all pregnant women in China $(2,3)$. GDM is associated with several adverse events, including stillbirth, fetal macrosomia and development of type 2 DM later in life (4-6). The activation of inflammation in placenta and adipose tissue plays key roles in IR during the pathogenesis of GDM $(7,8)$. Several inflammatory cytokines derived from placenta and adipose tissue participate in the activation of inflammation and initiate or aggravate IR during pregnancy (9-11). The placenta is a highly specialized organ during pregnancy that releases various cytokines and hormones, and contributes to the maternal IR (12-14). Since IR significantly improves immediately after delivery in GDM women $(15,16)$, it is speculated that the placenta may play a key role in the activation of inflammation and initiation of IR during GDM pathogenesis. However, the mechanism responsible for the regulation of inflammation in GDM placenta remains unclear.

Interleukin (IL)-1 $\beta$ and IL-18 are important inflammatory cytokines in the initiation of maternal IR during GDM (17-19). The animal experimental study by Schulze et al (20) reported that treatment with an anti-IL-1 $\beta$ antibody improved glucose-tolerance of GDM mice. The nucleotide binding and oligomerization domain-like receptor family pyrin domain-containing 3 (NLRP3) inflammasome participates in the regulation of IL-1 $\beta$ and IL-18 production $(21,22)$. The NLRP3 inflammasome can be activated by a wide range of pathogens and cellular damages, resulting in the generation of cleaved caspase-1, and produces IL-1 $\beta$ and IL-18 $(21,22)$. 
Previous studies have demonstrated that activation of the NLRP3 inflammasome is significantly elevated in patients with obesity, dyslipidemia and diabetes (23-25). According to the animal experimental study by Zhang et al (26), the expression levels of NLRP3 and caspase-1 are elevated in the placenta tissues of GDM mice. However, given that the expression of the NLRP3 inflammasome has not yet been investigated in clinical GDM placenta samples, further studies are required to determine the mechanism of the excessive activation of the NLPR3 inflammasome in placenta of GDM.

Known as 'the third endogenous gaseous signaling transmitter', hydrogen sulfide $\left(\mathrm{H}_{2} \mathrm{~S}\right)$ exerts biological functions, including anti-inflammatory, anti-oxidative stress and anti-apoptosis $(27,28)$. Our previous study demonstrated that $\mathrm{H}_{2} \mathrm{~S}$ suppresses activation of the NLPR3 inflammasome in adipocytes (29). Teng et al (30) reported that $\mathrm{H}_{2} \mathrm{~S}$ concentration significantly decreases in parturient women with GDM, suggesting that decreasing $\mathrm{H}_{2} \mathrm{~S}$ may be involved in the pathogenesis of GDM. $\mathrm{H}_{2} \mathrm{~S}$ is synthesized by L-cysteine in a range of mammalian tissues mainly by cystathionine- $\gamma$-lyase (CSE) and cystathionine- $\beta$-synthetase (CBS) (31). Our previous study demonstrated that human placenta samples express $\mathrm{H}_{2} \mathrm{~S}$ synthetase, CSE and CBS, and deficiency of CSE and CBS in the placenta is associated with preeclampsia (32). Previous studies have also reported that deficiency in $\mathrm{H}_{2} \mathrm{~S}$ synthetase is associated with other pregnancy complications, including premature labor (33) and fetal growth restriction (34). Thus, $\mathrm{H}_{2} \mathrm{~S}$ may participate in the pathogenesis of GDM by regulating activation of the NLPR3 inflammasome in placentas. The present study aimed to investigate the expression of the NLPR3 inflammasome and $\mathrm{H}_{2} \mathrm{~S}$ synthetases, CSE and CBS in clinical GDM placenta samples. In addition, the regulatory effect of $\mathrm{H}_{2} \mathrm{~S}$ on the NLPR3 inflammasome in the cultured extravillous trophoblast cell line, HTR-8/SVneo was investigated.

\section{Materials and methods}

Clinical samples. Human placenta tissues were collected from pregnant women with GDM $(n=16)$ and healthy pregnant women at term $(n=16)$ who underwent elective cesarean section between January 2019 and December 2020 at the Chinese PLA 903rd Hospital and Women's Hospital School of Medicine Zhejiang University. The clinical characteristics of the pregnant women are presented in Table I. The present study was approved by the Medical Ethics Committee of the Chinese PLA 903rd Hospital (ethics approval data and no. 2017/03/05) and written informed consent was provided by all participants prior to the study start. Clinical placenta samples were collected within 30 min of cesarean birth, and three small pieces of tissues from separate lobules were randomly taken from each placenta. The tissues were washed with normal saline, immediately frozen in liquid nitrogen and subsequently stored at $-80^{\circ} \mathrm{C}$ until subsequent experimentation.

Human placental cell culture and treatment. The human first trimester extravillous trophoblast cell line, HTR-8/SVneo was gifted by Professor Xin Ni at the Research Center for Molecular Metabolomics, Xiangya Hospital Central. Cells were recovered and incubated in RPMI-1640 media supplemented with $10 \%$ fetal bovine serum (both purchased from
Gibco; Thermo Fisher Scientific, Inc.) at $37^{\circ} \mathrm{C}$ with $5 \% \mathrm{CO}_{2}$ and $95 \%$ air, until they reached $\sim 90 \%$ confluence.

Cells were subsequently digested with $0.25 \%$ trypsin. Subsequently, $1 \times 10^{5}$ cells seeded into 12 -wells plates. To investigate the role of $\mathrm{H}_{2} \mathrm{~S}$ in regulating the NLPR3 inflammasome, cells were treated with different concentrations of NaHS (0, 10, 25 and 50 nmol/1; (Sigma-Aldrich; Merck KGaA) or L-cysteine (0, 0.25, 0.50 and $1.00 \mathrm{mmol} / \mathrm{l}$; Sigma-Aldrich; Merck KGaA) for $24 \mathrm{~h}$. The present study also investigated the role of the NLPR3 inflammasome in the production of IL-1 $\beta$ and IL-18, using the NLPR3 inflammasome inhibitor, $\mathrm{N}$-acetyl-tyrosyl-valyl-alanyl-aspartyl chloromethyl ketone (Ac-YVAD-CMK; Sigma-Aldrich; Merck KGaA).

Western blotting. Placental tissues ( 30-40 mg) were homogenized using RIPA lysis buffer (Beyotime Institute of Biotechnology) containing protease inhibitor cocktail tablet (Roche Diagnostics). Cultured human placental cells were scraped off the plate using RIPA lysis buffer containing protease inhibitor cocktail tablet (Roche Diagnostics). The lysates were subsequently centrifuged in the speed of $12,000 \mathrm{x} \mathrm{g}$ at $4^{\circ} \mathrm{C}$ for $15 \mathrm{~min}$ and the supernatant was collected. The concentration of protein in the supernatant was determined using the BCA kit (Beyotime Institute of Biotechnology). According to the concentration of protein, samples containing $30 \mu \mathrm{g}$ of protein were used for western blot analysis. The protein samples were separated via 4 and 10\% SDS-PAGE, transferred onto nitrocellulose membranes and blocked by $5 \%$ skim milk at room temperature for $2 \mathrm{~h}$. The membranes were incubated with primary antibodies against NLRP3 (1:1,000; ab263899; Abcam), cleaved caspase-1 (1:1,000; ab179515; Abcam) and $\beta$-actin (1:8,000; cat. no. A5441; Sigma-Aldrich; Merck KGaA) overnight at $4^{\circ} \mathrm{C}$. Following the primary incubation, membranes were incubated with a goat anti-rabbit secondary HRP-conjugated antibody (1:5,000; cat. no. BA1054; Wuhan Boster Biological Technology, Ltd.) at room temperature for $1 \mathrm{~h}$. Protein bands were visualized using the enhanced chemiluminescence substrate kit (Merck KGaA) and ChemiScope 6000EXP and the band intensities were calculated by ImageJ (version 1.51b; National Institutes of Health). Then ratio of band intensities to $\beta$-actin was obtained to quantify the relative protein expression levels.

ELISA. Following treatment, the culture media of the human placental cells was collected and IL-1 $\beta$ and IL-18 production was determined using the IL-1 $\beta$ ELISA kit (cat. no. F10770) and IL-18 ELISA kit (cat. no. F10920) (both Shanghai Westang Biotech), according to the manufacturer's instructions. All experiments were performed in duplicate.

Statistical analysis. Data are presented as the mean \pm SEM in SPSS (version 20; IBM Corp.). Each experiment in HTR-8/SVneo was repeated four times. All data were tested for homogeneity of variance using the Bartlett's test before analyzing the significance. Unpaired Student's t-test was used to compare differences between two groups, while one-way ANOVA followed by Bonferroni's post hoc test was used to compare differences between multiple groups. Pearson's analysis was used to analyze the correlation between two indexes. $\mathrm{P}<0.05$ was considered to indicate a statistically significant difference. 
Table I. Clinical characteristics of the pregnant women enrolled in the present study.

\begin{tabular}{|c|c|c|c|}
\hline Clinical characteristic & Normal pregnant women $(n=16)$ & GDM pregnant women $(n=16)$ & P-value \\
\hline Maternal age, years & $31.10 \pm 3.28$ & $32.42 \pm 4.51$ & 0.300 \\
\hline Gestational age, week & $38.60 \pm 0.94$ & $38.05 \pm 1.22$ & 0.125 \\
\hline $\mathrm{BMI}, \mathrm{kg} / \mathrm{m}^{2}$ & $26.75 \pm 1.75$ & $27.60 \pm 3.34$ & 0.326 \\
\hline \multicolumn{4}{|l|}{ Blood pressure, $\mathrm{mmHg}$} \\
\hline Systolic & $116.05 \pm 11.17$ & $117.58 \pm 10.65$ & 0.665 \\
\hline Diastolic & $73.45 \pm 9.67$ & $73.37 \pm 9.17$ & 0.979 \\
\hline \multicolumn{4}{|l|}{ Blood glucose, mmol/l } \\
\hline OGTT $0 \mathrm{~h}$ & $4.46 \pm 0.31$ & $4.88 \pm 0.56$ & $0.043^{\mathrm{a}}$ \\
\hline OGTT $1 \mathrm{~h}$ & $6.59 \pm 1.13$ & $10.94 \pm 2.12$ & $<0.001^{\mathrm{b}}$ \\
\hline OGTT 2 h & $5.89 \pm 0.75$ & $9.66 \pm 1.92$ & $<0.001^{\mathrm{b}}$ \\
\hline $\mathrm{HbA} 1 \mathrm{c}, \%$ & $4.95 \pm 0.27$ & $5.28 \pm 0.45$ & $0.014^{\mathrm{a}}$ \\
\hline Infant birth weight, g & $3407.75 \pm 349.45$ & $3315.79 \pm 417.34$ & 0.459 \\
\hline
\end{tabular}

${ }^{\mathrm{a}} \mathrm{P}<0.05 ;{ }^{\mathrm{b}} \mathrm{P}<0.01$. GDM, gestational diabetes mellitus; BMI, body mass index.
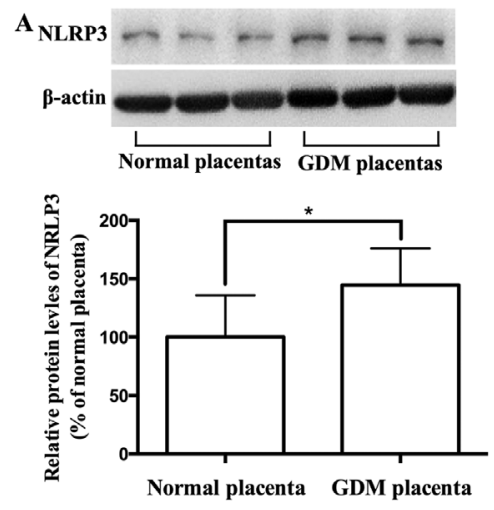

C

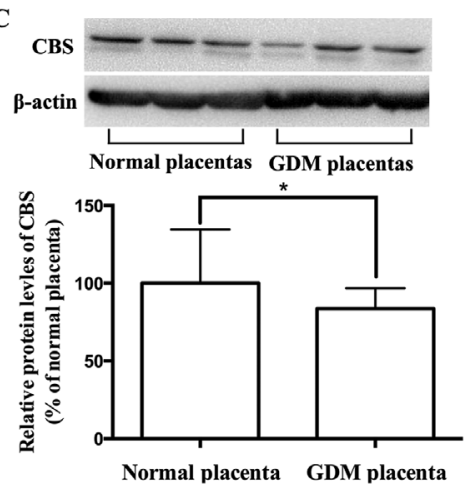

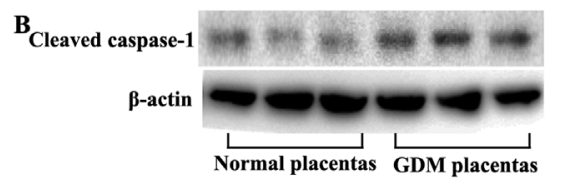

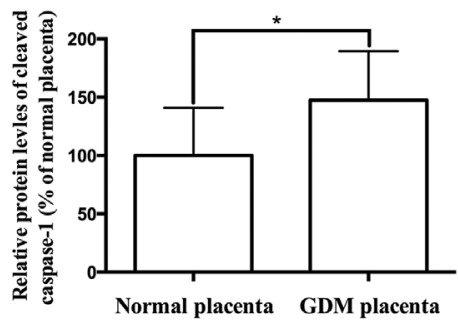

D
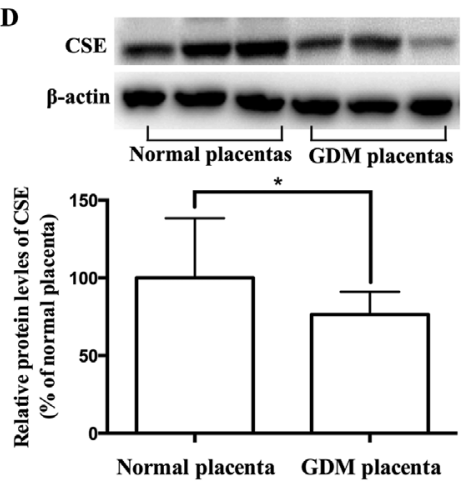

Figure 1. Comparison of NLRP3, cleaved caspase-1, CBS and CSE expression levels between healthy placentas and GDM placentas. (A) Western blot analysis was performed to detect the protein expression levels of (A) NLRP3, (B) cleaved caspase-1, (C) CBS and (D) CSE in healthy placentas and GDM placentas. Representative protein bands are presented on top of the corresponding histogram. Data are presented as the mean \pm SEM. ${ }^{*}<0.05$. NLRP3, NLR family pyrin domain containing-3; CBS, cystathionine- $\beta$-synthetase; CSE, synthetase cystathionine- $\gamma$-lyase; GDM, gestational diabetes mellitus.

\section{Results}

Expression levels of NLRP3, cleaved caspase-1, CBS and CSE in GDM and healthy placentas. To investigate the role of $\mathrm{H}_{2} \mathrm{~S}$ in the excessive activation of the NLPR3 inflammasome in GDM placenta, the expression levels of NLRP3, cleaved caspase-1, and the $\mathrm{H}_{2} \mathrm{~S}$ synthetases CBS and CSE in placentas were determined. As presented in Fig. 1A-D, the expression levels of NLRP3 and cleaved caspase-1 increased, while the expression levels of CBS and CSE decreased in GDM placentas compared with healthy placentas. The correlation between NLRP3 and cleaved caspase- 1 with the $\mathrm{H}_{2} \mathrm{~S}$ synthetases were analyzed. As presented in Fig. 2A-D, the levels of CBS and CSE were inversely correlated with NLRP3 and cleaved caspase-1 in GDM placentas.

Effect of $\mathrm{H}_{2} \mathrm{~S}$ on the expression of the NLPR3 inflammasome in placental cells. Our previous study demonstrated that the expression of the NLPR3 inflammasome decreases via $\mathrm{H}_{2} \mathrm{~S}$ in 

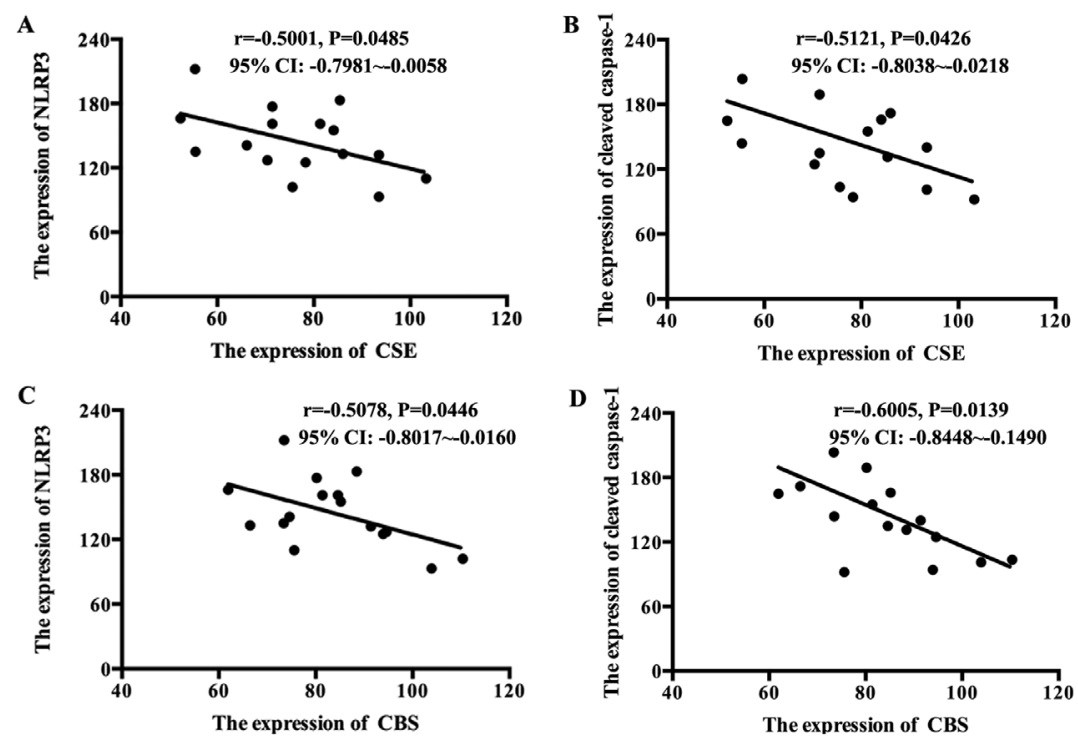

Figure 2. Correlation between NLRP3, cleaved caspase-1 and CBS and CSE in GDM placentas. Pearson's correlation analysis was performed to determine the correlation between NLRP3, cleaved caspase-1 and CBS and CSE in GDM placentas. (A) CSE was inversely correlated with NLRP3 in GDM placentas. (B) CSE was inversely correlated with cleaved caspase-1 in GDM placentas. (C) CBS was inversely correlated with NLRP3 in GDM placentas. (D) CBS was inversely correlated with cleaved caspase-1 in GDM placentas. NLRP3, NLR family pyrin domain containing-3; CBS, cystathionine- $\beta$-synthetase; CSE, synthetase cystathionine- $\gamma$-lyase; GDM, gestational diabetes mellitus.

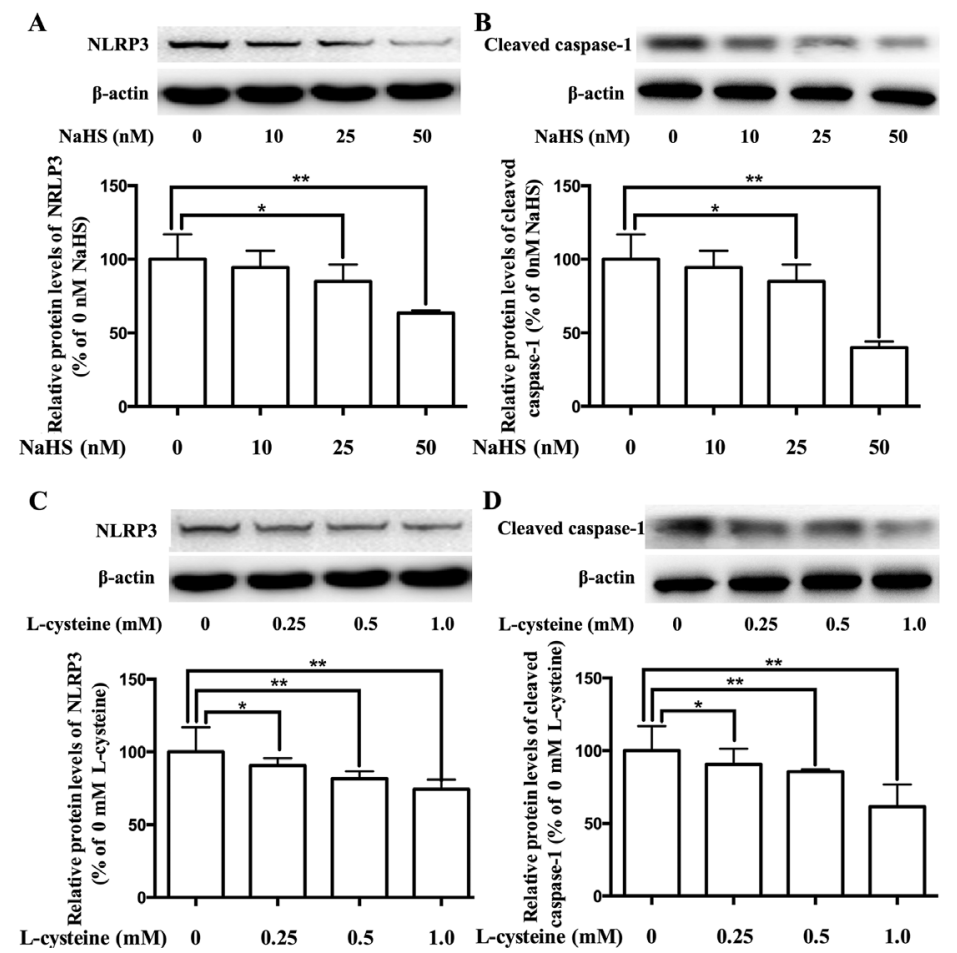

Figure 3. Effects of NaHS and L-cysteine on the expression levels of NLRP3 and cleaved caspase-1 in human placental cells. Western blot analysis was performed to detect the protein expression levels of NLRP3 and cleaved caspase-1 in cells. NaHS regulated the expression levels of (A) NLRP3 and (B) cleaved caspase-1 in human placental cells. L-cysteine regulated the expression levels of (C) NLRP3 and (D) cleaved caspase-1 in human placental cells. Representative protein bands are presented on top of the corresponding histogram. Data are presented as the mean \pm SEM $(n=4)$. " $P<0.05$ and ${ }^{* *} \mathrm{P}<0.01$. NLRP3, NLR family pyrin domain containing-3.

adipocytes (29). To investigate the role of $\mathrm{H}_{2} \mathrm{~S}$ in the regulation of the NLPR3 inflammasome in placenta, placental cells were cultured and treated with $\mathrm{H}_{2} \mathrm{~S}$ donor $\mathrm{NaHS}$ or $\mathrm{H}_{2} \mathrm{~S}$ precursor L-cysteine. As presented in Fig. 3A-D, treatment with NaHS and L-cysteine significantly inhibited the expression levels of NLRP3 and cleaved caspase-1, in dose-dependent manners.
Effect of $\mathrm{H}_{2} \mathrm{~S}$ on the production of IL-1 $\beta$ and IL-18 in placental cells. Activation of the NLPR3 inflammasome releases IL-1 $\beta$ and IL-18 $(21,22)$. To confirm the role of $\mathrm{H}_{2} \mathrm{~S}$ in the regulation of the NLPR3 inflammasome in placenta, the contents of IL-1 $\beta$ and IL-18 in the culture media of placental cells were determined. As presented in Fig. 4, treatment with NaHS and 

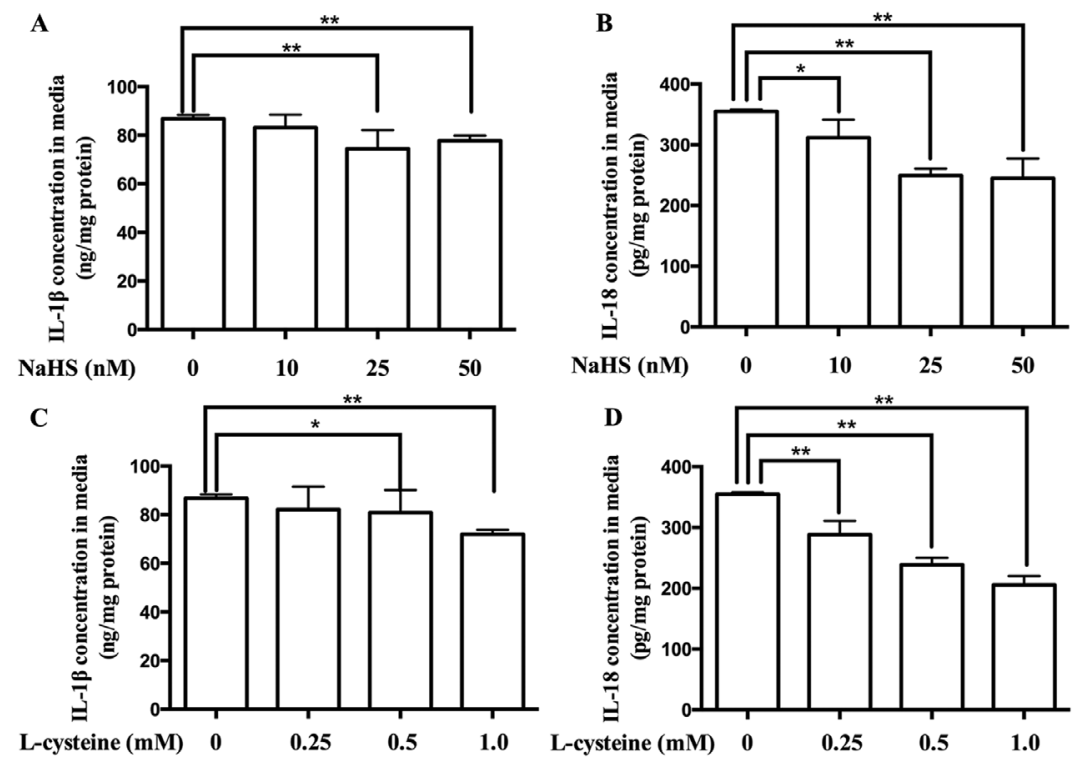

Figure 4. Effects of NaHS and L-cysteine on the contents of IL-1 $\beta$ and IL-18 in the culture media of human placental cells. ELISA was performed to determine IL-1 $\beta$ and IL-18 contents. NaHS regulated the contents of (A) IL-1 $\beta$ and (B) IL-18 in the culture media. L-cysteine regulated the contents of (C) IL-1 $\beta$ and (D) IL-18 in the culture media. Data are presented as the mean $\pm \operatorname{SEM}(n=4) .{ }^{*} \mathrm{P}<0.05$ and ${ }^{* *} \mathrm{P}<0.01$. IL, interleukin.
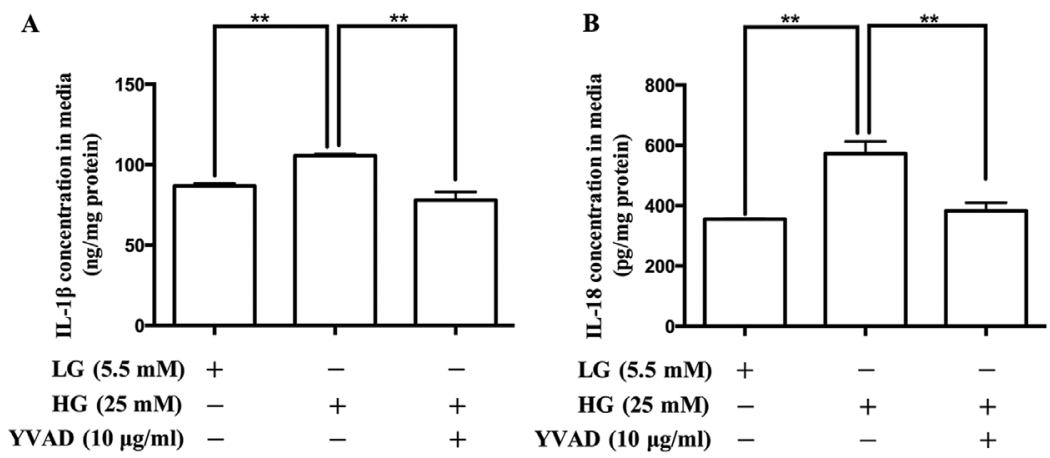

Figure 5. Effects of the NLRP3 inflammasome inhibitor, Ac-YVAD-CMK on the contents of IL-1 $\beta$ and IL-18 in the culture media of human placental cells. ELISA was performed to determine the contents of IL-1 $\beta$ and IL-18 in the culture media. Ac-YVAD-CMK regulated the contents of (A) IL- $1 \beta$ and (B) IL-18 in the culture media. Data are presented as the mean \pm SEM $(n=4) .{ }^{* *} \mathrm{P}<0.01$. NLR family pyrin domain containing-3; Ac-YVAD-CMK, N-acetyl-tyrosyl-valyl-alanyl-aspartyl chloromethyl ketone; IL, interleukin; LG, low glucose; HG, high glucose.

L-cysteine decreased the production of IL-1 $\beta$ and IL-18, in dose-dependent manners.

Effect of the NLRP3 inflammasome inhibitor on the production of IL-1 $\beta$ and IL-18 in placental cells. To confirm the role of the NLRP3 inflammasome in the production of IL-1 $\beta$ and IL-18 in placental cells, the NLRP3 inflammasome inhibitor, Ac-YVAD-CMK was used. As presented in Fig. 5, treatment with Ac-YVAD-CMK decreased the release of IL-1 $\beta$ and IL-18.

\section{Discussion}

The results of the present study demonstrated that the reduced expression of $\mathrm{H}_{2} \mathrm{~S}$ synthetases, CSE and CBS was correlated with the excessive activation of the NLPR3 inflammasome in GDM placenta. $\mathrm{H}_{2} \mathrm{~S}$ significantly suppressed the activation of the NLPR3 inflammasome in human placental cells in vitro. Furthermore, the NLPR3 inflammasome was involved in the production of IL-1 $\beta$ and IL-18 in human placental cells.
Known as a highly specialized organ during pregnancy, the placenta serves as the interface between maternal and fetal circulation (35). In recent years, the key role of the placenta in the occurrence and development of GDM has been reported by multiple studies (36-38). Currently, IR is the critical pathophysiological characteristic of GDM, which is also found during normal pregnancy. Placenta derived hormones, cytokines and gaseous signaling transmitter can induce IR by interfering with insulin receptor signal transduction (12-14). Furthermore, the dysregulation of hormones, cytokines and gaseous signaling transmitter in placenta may aggravate IR and trigger abnormal glucose metabolism (12-14). Thus, the present study investigated the key molecules in the placenta responsible for the pathogenesis of GDM.

The overactive inflammatory response may be the initiating factor for IR. Cytokines of the IL-1 family critically regulate the inflammatory response by controlling several inflammation processes $(39,40)$. Both IL-1 $\beta$ and IL-18, which are classic pro-inflammatory cytokines of the IL-1 family, participate in the initiation of IR of GDM and type 2 DM (17-19). The 
production of IL-1 $\beta$ and IL-18 is regulated by the NLRP3 inflammasome in different types of tissues and cells. The NLRP3 inflammasome complex is composed of NLRP3, ASC and pro caspase-1. Activation of the inflammasome recruits and cleaves pro caspase-1, which results in the formation of cleaved caspase-1. Subsequently, cleaved caspase- 1 converts pro-IL-1 $\beta$ and pro-IL-18 into the mature forms, IL-1 $\beta$ and IL-18 $(21,22)$. According to the animal experimental study by Zhang et al (26), the expression levels of NLRP3 and cleaved caspase-1 are elevated in the placenta tissues of GDM mice. The results of the present study demonstrated that the expression levels of NLRP3 and cleaved caspase-1 were elevated in the clinical placenta samples collected from pregnant women with GDM. Taken together, the results of the present study suggest that excessive activation of the NLRP3 inflammasome in the placenta may be involved in the development of GDM.

Further research on the mechanism of the regulation of the NLRP3 inflammasome in the placenta is required. $\mathrm{H}_{2} \mathrm{~S}$ is a lately identified gaseous signaling transmitter that mediates a variety of biological activities, including, anti-apoptotic and anti-oxidative stress $(27,28)$. During pregnancy, the abnormal production of $\mathrm{H}_{2} \mathrm{~S}$ and the dysregulation of the $\mathrm{H}_{2} \mathrm{~S}$ synthetases, CBS and CSE are associated with various pregnancy complications $(32,41,42)$. The results of the present study demonstrated that the expression of the $\mathrm{H}_{2} \mathrm{~S}$ synthetases, CBS and CSE were significantly downregulated in GDM placenta samples, which was consistent with the findings reported by Teng et al (30). Our previous study investigated the regulatory effect of $\mathrm{H}_{2} \mathrm{~S}$ on the NLRP3 inflammasome in the pathogenesis of vascular complications of type $2 \mathrm{DM}$, and the results demonstrated that $\mathrm{H}_{2} \mathrm{~S}$ significantly suppressed activation of the NLRP3 inflammasome in adipocyte (23). Other studies have also reported the role of $\mathrm{H}_{2} \mathrm{~S}$ in regulating the NLRP3 inflammasome. For example, Jia et al (43), Zheng et al (44) and Su et al (45) reported the inhibitory effect of $\mathrm{H}_{2} \mathrm{~S}$ on the NLRP3 inflammasome in diabetic myocardial injury model, diabetes-accelerated atherosclerosis model and renal injury model.

The results of the present study demonstrated an inverse correlation between the $\mathrm{H}_{2} \mathrm{~S}$ synthetases and the NLRP3 inflammasome in GDM placentas, suggesting that $\mathrm{H}_{2} \mathrm{~S}$ may participate in regulating the NLRP3 inflammasome in placenta. The effect of $\mathrm{H}_{2} \mathrm{~S}$ on the NLRP3 inflammasome in vitro was also investigated. In human placental cells, both the $\mathrm{H}_{2} \mathrm{~S}$ donor and precursor decreased the expression levels of NLRP3 and cleaved caspase-1, as well as the production of IL-1 $\beta$ and IL-18. In addition, the NLRP3 inflammasome inhibitor decreased the production of IL-1 $\beta$ and IL-18 in human placental cells. Collectively, these results suggest that $\mathrm{H}_{2} \mathrm{~S}$ plays a regulatory role in the activation of the NLRP3 inflammasome, and $\mathrm{H}_{2} \mathrm{~S}$ synthetase deficiency results in excessive activation of the NLRP3 inflammasome and excessive production of IL-1 $\beta$ and IL-18 in GDM placenta.

Most previous studies focused on the downstream biological effects of $\mathrm{H}_{2} \mathrm{~S}(29,32,43-45)$; however, the mechanism responsible for the upstream regulatory factor for the expression of CBS and CSE, and the production of $\mathrm{H}_{2} \mathrm{~S}$ remains unclear. Recently, several studies investigated the upstream regulatory mechanism for the expression of CBS and CSE, and the production of $\mathrm{H}_{2} \mathrm{~S}$, and the results demonstrated that high fat $(46,47)$, high salt (48), hypoxia (49) and oxidative stress (50) inhibited the expression of
CBS and CSE, and the production of $\mathrm{H}_{2} \mathrm{~S}$. Conversely, vitamin D supplementation increased CSE expression and the production of $\mathrm{H}_{2} \mathrm{~S}$ (51). Other clinical studies have reported that high-fat and high-salt diet, vitamin D deficiency during pregnancy (52-54), hypoxia and oxidative stress in the placenta $(55,56)$ are associated with the pathogenesis of GDM. Taken together, these results suggest that high-fat and high-salt diet, vitamin D, hypoxia and oxidative stress may be upstream regulatory factors for the expression of CBS and CSE, and the production of $\mathrm{H}_{2} \mathrm{~S}$ in GDM. However, further studies are required to determine the specific mechanism responsible for the expression of CBS and CSE, and the production of $\mathrm{H}_{2} \mathrm{~S}$ in GDM.

In conclusion, the results of the present study demonstrated the role of the NLRP3 inflammasome and $\mathrm{H}_{2} \mathrm{~S}$ in the occurrence and development of GDM. Excessive activation of the NLRP3 inflammasome may be induced by the $\mathrm{H}_{2} \mathrm{~S}$ synthetase deficiency in the placenta, and activation of the NLRP3 inflammasome mediates the elevated production of IL-1 $\beta$ and IL-18, thus initiating maternal IR and causing abnormal glucose metabolism.

\section{Acknowledgements}

The authors of the present study would like to thank Professor Xin Ni (Research Center for Molecular Metabolomics, Xiangya Hospital Central, Changsha, Hunan 410008 , China) for valuable comments on the manuscript.

\section{Funding}

The present study was supported by the Natural Science Foundation of China (grant no. 81701481) and the Medical and Health Science and Technology Project of Zhejiang Province (grant no. 2019RC253).

\section{Availability of data and materials}

The datasets used and analyzed during the current study are available from the corresponding author on reasonable request.

\section{Authors' contributions}

TXH and XBD were involved in the overall structuring and designing of the study, drafting and revising the manuscript and obtaining funding. WW and QYT contributed to the major cell experiments, including cell culture, protein expression and cytokine contents determination. FFX and QL collected the clinical samples and data. YR and JW contributed to the analysis of data. All authors reviewed the initial manuscript and revised it critically for important intellectual content. All authors have confirmed the authenticity of all the raw data and read and approved the final manuscript.

\section{Ethics approval and consent to participate}

The present study was approved by the Medical Ethics Committee of the Chinese PLA 903rd Hospital (ethics approval data and no. 2017/03/05) and performed in accordance with the 1964 Helsinki declaration and its later amendments or comparable ethical standards. Written informed consent was provided by all participants prior to the study start. 


\section{Patient consent for publication}

Not applicable.

\section{Competing interests}

The authors declare that they have no competing interests.

\section{References}

1. Catalano PM: Trying to understand gestational diabetes. Diabet Med 31: 273-281, 2014.

2. Yan B, Yu Y, Lin M, Li Z, Wang L, Huang P, Song H, Shi X, Yang S, Li X, et al: High, but stable, trend in the prevalence of gestational diabetes mellitus: A population-based study in Xiamen, China. J Diabetes Investig 10: 1358-1364, 2019.

3. Wang M, Hu RY, Gong WW, Pan J, Fei FR, Wang H, Zhou XY Zhong JM and $\mathrm{Yu}$ M: Trends in prevalence of gestational diabetes mellitus in Zhejiang Province, China, 2016-2018. Nutr Metab (Lond) 18: 12, 2021.

4. Gualdani E, Di Cianni G, Seghieri M, Francesconi P and Seghieri G: Pregnancy outcomes and maternal characteristics in women with pregestational and gestational diabetes: A retrospective study on 206,917 singleton live births. Acta Diabetol 58: 1169-1176, 2021.

5. de Sousa RAL, de Lima EV, da Silva TP, de Souza RV, Figueiredo CP, Passos GF and Clarke JR: Late Cognitive Consequences of Gestational Diabetes to the Offspring, in a New Mouse Model. Mol Neurobiol 56: 7754-7764, 2019.

6. Kajantie E, Osmond C and Eriksson JG: Gestational hypertension is associated with increased risk of type 2 diabetes in adult offspring: the Helsinki Birth Cohort Study. Am J Obstet Gynecol 216: 281 e281-281 e287, 2017.

7. Cinkajzlová A, Anderlová K, Simják P, Lacinová Z, Kloučková J, Kratochvílová H, Krejčí H, Pařízek A, Mráz M, Kršek M, et al: Subclinical Inflammation and Adipose Tissue Lymphocytes in Pregnant Females With Gestational Diabetes Mellitus. J Clin Endocrinol Metab 105: dgaa528, 2020.

8. Olmos-Ortiz A, Flores-Espinosa P, Díaz L, Velázquez P, Ramírez-Isarraraz $\mathrm{C}$ and Zaga-Clavellina V: Immunoendocrine Dysregulation during Gestational Diabetes Mellitus: The Central Role of the Placenta. Int J Mol Sci 22: 8087, 2021.

9. Rancourt RC, Ott R, Ziska T, Schellong K, Melchior K, Henrich W and Plagemann A: Visceral Adipose Tissue Inflammatory Factors (TNF-Alpha, SOCS3) in Gestational Diabetes (GDM) Epigenetics as a Clue in GDM Pathophysiology. Int J Mol Sci 21 : 479, 2020.

10. Keckstein S, Pritz S, Amann N, Meister S, Beyer S, Jegen M, Kuhn C, Hutter S, Knabl J, Mahner S, et al: Sex Specific Expression of Interleukin 7, 8 and 15 in Placentas of Women with Gestational Diabetes. Int J Mol Sci 21: 8026, 2020.

11. Tsiotra PC,Halvatsiotis P,Patsouras K, Maratou E, Salamalekis G, Raptis SA, Dimitriadis G and Boutati E: Circulating adipokines and mRNA expression in adipose tissue and the placenta in women with gestational diabetes mellitus. Peptides 101: 157-166, 2018.

12. Simpson S, Smith L and Bowe J: Placental peptides regulating islet adaptation to pregnancy: Clinical potential in gestational diabetes mellitus. Curr Opin Pharmacol 43: 59-65, 2018.

13. Hill DJ: Placental control of metabolic adaptations in the mother for an optimal pregnancy outcome. What goes wrong in gestational diabetes? Placenta 69: 162-168, 2018.

14. Ngala RA, Fondjo LA, Gmagna P, Ghartey FN and Awe MA: Placental peptides metabolism and maternal factors as predictors of risk of gestational diabetes in pregnant women. A case-control study. PLoS One 12: e0181613, 2017.

15. Skajaa GO, Fuglsang J, Knorr S, Møller N, Ovesen P and Kampmann U: Changes in insulin sensitivity and insulin secretion during pregnancy and post partum in women with gestational diabetes. BMJ Open Diabetes Res Care 8: e001728, 2020 .

16. Waters TP, Kim SY, Sharma AJ, Schnellinger P, Bobo JK, Woodruff RT, Cubbins LA, Haghiac M, Minium J, Presley L, et al: Longitudinal changes in glucose metabolism in women with gestational diabetes, from late pregnancy to the postpartum period. Diabetologia 63: 385-394, 2020
17. Liu T, Deng JM, Liu YL, Chang L and Jiang YM: The relationship between gestational diabetes mellitus and interleukin 1beta gene polymorphisms in southwest of China. Medicine (Baltimore) 99: e22679, 2020

18. Fatima SS, Alam F, Chaudhry B and Khan TA: Elevated levels of chemerin, leptin, and interleukin-18 in gestational diabetes mellitus. J Matern Fetal Neonatal Med 30: 1023-1028, 2017.

19. Gomes CP, Torloni MR, Gueuvoghlanian-Silva BY, Alexandre SM, Mattar R and Daher S: Cytokine levels in gestational diabetes mellitus: A systematic review of the literature. Am J Reprod Immunol 69: 545-557, 2013.

20. Schulze F, Wehner J, Kratschmar DV, Makshana V, Meier DT, Häuselmann SP, Dalmas E, Thienel C, Dror E, Wiedemann SJ, et al: Inhibition of IL-1beta improves Glycaemia in a Mouse Model for Gestational Diabetes. Sci Rep 10: 3035, 2020.

21. Zhou F, Li C and Zhang SY: NLRP3 inflammasome: A new therapeutic target for high-risk reproductive disorders? Chin Med J (Engl) 134: 20-27, 2020.

22. Fang X, Wang Y, Zhang Y, Li Y, Kwak-Kim J and Wu L: NLRP3 Inflammasome and Its Critical Role in Gynecological Disorders and Obstetrical Complications. Front Immunol 11: 555826, 2021.

23. Gora IM, Ciechanowska A and Ladyzynski P: NLRP3 Inflammasome at the Interface of Inflammation, Endothelial Dysfunction, and Type 2 Diabetes. Cells 10: 10, 2021.

24. Yu ZW, Zhang J, Li X, Wang Y, Fu YH and Gao XY: A new research hot spot: The role of NLRP3 inflammasome activation, a key step in pyroptosis, in diabetes and diabetic complications. Life Sci 240: 117138, 2020.

25. Mastrocola R, Aragno M, Alloatti G, Collino M, Penna C and Pagliaro P: Metaflammation: Tissue-Specific Alterations of the NLRP3 Inflammasome Platform in Metabolic Syndrome. Curr Med Chem 25: 1294-1310, 2018.

26. Zhang R, Zhang X, Xing B, Zhao J, Zhang P, Shi D and Yang F: Astragaloside IV attenuates gestational diabetes mellitus via targeting NLRP3 inflammasome in genetic mice. Reprod Biol Endocrinol 17: 77, 2019.

27. Chen CQ, Xin H and Zhu YZ: Hydrogen sulfide: Third gaseous transmitter, but with great pharmacological potential. Acta Pharmacol Sin 28: 1709-1716, 2007.

28. Tang C, Li X and Du J: Hydrogen sulfide as a new endogenous gaseous transmitter in the cardiovascular system. Curr Vasc Pharmacol 4: 17-22, 2006.

29. Hu TX, Zhang NN, Ruan Y, Tan QY and Wang J: Hydrogen sulfide modulates high glucose-induced NLRP3 inflammasome activation in 3T3-L1 adipocytes. Exp Ther Med 19: 771-776, 2020.

30. Teng Y, Xuan S, Jiang M, Tian L, Tian J and Chang Q: Expression of $\mathrm{H}_{2} \mathrm{~S}$ in Gestational Diabetes Mellitus and Correlation Analysis with Inflammatory Markers IL-6 and TNF- $\alpha$. J Diabetes Res 2020: 3085840, 2020.

31. Renga B: Hydrogen sulfide generation in mammals: The molecular biology of cystathionine- $\beta$ - synthase (CBS) and cystathionine- $\gamma$-lyase (CSE). Inflamm Allergy Drug Targets 10: 85-91, 2011

32. Hu T, Wang G, Zhu Z, Huang Y, Gu H and Ni X: Increased ADAM10 expression in preeclamptic placentas is associated with decreased expression of hydrogen sulfide production enzymes. Placenta 36: 947-950, 2015.

33. Sun Q, Chen Z, He P, Li Y, Ding X, Huang Y, Gu H and Ni X: Reduced Expression of Hydrogen Sulfide-Generating Enzymes Down-Regulates 15-Hydroxyprostaglandin Dehydrogenase in Chorion during Term and Preterm Labor. Am J Pathol 188: 63-71, 2018

34. Lu L, Kingdom J, Burton GJ and Cindrova-Davies T: Placental Stem Villus Arterial Remodeling Associated with Reduced Hydrogen Sulfide Synthesis Contributes to Human Fetal Growth Restriction. Am J Pathol 187: 908-920, 2017.

35. Ganguly E, Hula N, Spaans F, Cooke CM, Davidge ST and Ganguly E: Placenta-targeted treatment strategies: An opportunity to impact fetal development and improve offspring health later in life. Pharmacol Res 157: 104836, 2020.

36. Tsai K, Tullis B, Jensen T, Graff T, Reynolds P and Arroyo J: Differential expression of mTOR related molecules in the placenta from gestational diabetes mellitus (GDM), intrauterine growth restriction (IUGR) and preeclampsia patients. Reprod Biol 21: 100503, 2021.

37. Awamleh Z, Butcher DT, Hanley A, Retnakaran R, Haertle L, Haaf T, Hamilton J and Weksberg R: Exposure to Gestational Diabetes Mellitus (GDM) alters DNA methylation in placenta and fetal cord blood. Diabetes Res Clin Pract 174: 108690, 2021. 
38. Sarina, Li DF, Feng ZQ, Du J, Zhao WH, Huang N, Jia JC, Wu ZY, Alamusi, Wang YY, Ji XL and Yu L: Mechanism of Placenta Damage in Gestational Diabetes Mellitus by Investigating TXNIP of Patient Samples and Gene Functional Research in Cell Line. Diabetes Ther 10: 2265-2288, 2019.

39. Dinarello CA: Overview of the IL-1 family in innate inflammation and acquired immunity. Immunol Rev 281: 8-27, 2018.

40. Ballak DB, Stienstra R, Tack CJ, Dinarello CA and van Diepen JA IL-1 family members in the pathogenesis and treatment of metabolic disease: Focus on adipose tissue inflammation and insulin resistance. Cytokine 75: 280-290, 2015.

41. Zochio GP, Possomato-Vieira JS, Chimini JS, da Silva MLS and Dias-Junior CA: Effects of fast versus slow-releasing hydrogen sulfide donors in hypertension in pregnancy and fetoplacenta growth restriction. Naunyn Schmiedebergs Arch Pharmacol 392: $1561-1568,2019$.

42. You X, Chen Z, Zhao H, Xu C, Liu W, Sun Q, He P, Gu H and Ni X: Endogenous hydrogen sulfide contributes to uterine quiescence during pregnancy. Reproduction 153: 535-543, 2017.

43. Jia Q, Mehmood S, Liu X, Ma S and Yang R: Hydrogen sulfide mitigates myocardial inflammation by inhibiting nucleotide-binding oligomerization domain-like receptor protein 3 inflammasome activation in diabetic rats. Exp Biol Med (Maywood) 245: 221-230, 2020.

44. Zheng Q, Pan L and Ji Y: H 2 S protects against diabetes-accelerated atherosclerosis by preventing the activation of NLRP3 inflammasome. J Biomed Res 34: 94-102, 2019.

45. Su Y, Wang Y, Liu M and Chen H: Hydrogen sulfide attenuates renal $\mathrm{I} / \mathrm{R}$-induced activation of the inflammatory response and apoptosis via regulating Nrf2-mediated NLRP3 signaling pathway inhibition. Mol Med Rep 24: 518, 2021.

46. Nguyen TTP, Kim DY, Lee YG, Lee YS, Truong XT, Lee JH, Song DK, Kwon TK, Park SH, Jung CH, et al: SREBP-1c impairs ULK1 sulfhydration-mediated autophagic flux to promote hepatic steatosis in high-fat-diet-fed mice. Mol Cell 81: 3820-3832.e7, 2021

47. Peh MT, Anwar AB, Ng DS, Atan MS, Kumar SD and Moore PK: Effect of feeding a high fat diet on hydrogen sulfide $\left(\mathrm{H}_{2} \mathrm{~S}\right)$ metabolism in the mouse. Nitric Oxide 41: 138-145, 2014

48. Moreira AM, Grisote SA, Francescato HDC, Coimbra TM, Elias LLK, Antunes-Rodrigues J and Ruginsk SG: Effects of endogenous $\mathrm{H}_{2} \mathrm{~S}$ production inhibition on the homeostatic responses induced by acute high-salt diet consumption. Mol Cell Biochem 476: 715-725, 2021.
49. Zheng W and Liu C: The cystathionine $\gamma$-lyase/hydrogen sulfide pathway mediates the trimetazidine-induced protection of $\mathrm{H} 9 \mathrm{c} 2$ cells against hypoxia/reoxygenation-induced apoptosis and oxidative stress. Anatol J Cardiol 22: 102-111, 2019.

50. Bibli SI and Fleming I: Oxidative Post-Translational Modifications: A Focus on Cysteine S-Sulfhydration and the Regulation of Endothelial Fitness. Antioxid Redox Signal: Sep 29, 2021 (Epub ahead of print). doi: 10.1089/ars.2021.0162.

51. Manna P and Jain SK: Vitamin D up-regulates glucose transporter 4 (GLUT4) translocation and glucose utilization mediated by cystathionine- $\gamma$-lyase (CSE) activation and $\mathrm{H}_{2} \mathrm{~S}$ formation in 3T3L1 adipocytes. J Biol Chem 287: 42324-42332, 2012.

52. Brown J, Alwan NA, West J, Brown S, McKinlay CJ, Farrar D and Crowther CA: Lifestyle interventions for the treatment of women with gestational diabetes. Cochrane Database Syst Rev 5: CD011970, 2017

53. Jiang XC, Liang ZD, Chen DL, Jia JP, Hu JR and Hu L: Correlation of Homocysteine, AHSG, CRP with Insulin Resistance, 25-(OH)2-VitD, Blood Lipids in Gestational Diabetes Patients. Clin Lab 67: 67, 2021.

54. Chen W, Li Y, Gao B, Li J, Zheng M and Chen X: Serum 25-hydroxyvitamin D levels in relation to lipids and clinical outcomes in pregnant women with gestational diabetes mellitus: An observational cohort study. BMJ Open 10: e039905, 2020.

55. Akarsu S, Bagirzade M, Omeroglu S and Büke B: Placental vascularization and apoptosis in Type- 1 and gestational DM. J Matern Fetal Neonatal Med 30: 1045-1050, 2017.

56. Kasture V, Sahay A and Joshi S: Cell death mechanisms and their roles in pregnancy related disorders. Adv Protein Chem Struct Biol 126: 195-225, 2021.

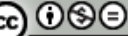

This work is licensed under a Creative Commons Attribution-NonCommercial-NoDerivatives 4.0 International (CC BY-NC-ND 4.0) License. 